\title{
Emergent Heavy Fermion Behavior at the Wigner-Mott Transition
}

\author{
Jaime Merino, ${ }^{1}$ Arnaud Ralko, ${ }^{2}$ and Simone Fratini ${ }^{2}$ \\ ${ }^{1}$ Departamento de Física Teórica de la Materia Condensada, Condensed Matter Physics Center (IFIMAC) \\ and Instituto Nicolás Cabrera, Universidad Autónoma de Madrid, Madrid 28049, Spain \\ ${ }^{2}$ Institut Néel-CNRS and Université Joseph Fourier, Boîte Postale 166, F-38042 Grenoble Cedex 9, France
}

(Received 9 April 2013; published 20 September 2013)

\begin{abstract}
We study charge ordering driven by Coulomb interactions on triangular lattices relevant to the Wigner-Mott transition in two dimensions. Dynamical mean-field theory reveals the pinball liquid phase, a charge ordered metallic phase containing quasilocalized (pins) coexisting with itinerant (balls) electrons. Based on an effective periodic Anderson model for this phase, we find an antiferromagnetic Kondo coupling between pins and balls and strong quasiparticle renormalization. Non-Fermi liquid behavior can occur in such charge ordered systems due to the spin-flip scattering of itinerant electrons off the pins in analogy with heavy fermion compounds.
\end{abstract}

The interplay between charge ordering $(\mathrm{CO})$ and the Mott metal-insulator transition (MIT) has common manifestations in narrow-band systems such as the transitionmetal oxides [1] (cuprates, manganites, nickelates, and cobaltates), low-dimensional organic conductors [2] as well as transition-metal dichalcogenides [3-5]. On the one hand, charge ordering phenomena are favored by the presence of electronic correlations, as these slow down the electronic motion and reduce the tendency to charge delocalization. Conversely, the reconstruction of the electronic spectrum upon entering a phase with broken symmetry can enhance the effects of correlations, extending the realm of Mott physics away from the usual integer fillings [2,6-13]. A fundamental relation between charge ordering and the Mott MIT has also been pointed out recently in the two-dimensional electron gas (2DEG), leading to the concept of the Wigner-Mott transition [8-11]. Close to the Wigner crystal phase induced by the long ranged Coulomb repulsion at low density, the electrons form an effectively narrow-band system at half-filling (one electron per site of the Wigner crystal [14]), whose physical properties resemble those of a Mott insulator. Correspondingly, it has been shown that the Wigner crystal melting shares several common features with the Mott transition $[8,9,15]$.

In the present Letter, we explore the interplay of charge order and electronic correlations starting from the extended Hubbard model on the triangular lattice, which frustrates the insulating $\mathrm{CO}$ found in bipartite lattices. We focus on the pinball liquid (PL) phase [16-18], i.e., a charge ordered metallic phase where quasilocalized electrons (pins) coexist with itinerant electrons (balls) over a broad range of concentrations. Applying the dynamical mean-field theory (DMFT) we find that the electron dynamics become heavily renormalized for a sufficiently large carrier concentration. The origin of this renormalization is elucidated via a mapping to a periodic Anderson model (PAM). The strong analogies with heavy fermions [19] indicate that non-Fermi liquid behavior can arise in correlated $\mathrm{CO}$ systems on frustrated lattices in a broad temperature range above the Kondo breakdown temperature scale.

Model.-The reference model to study the interplay between charge ordering and electronic correlations is the extended Hubbard model (EHM),

$H=-t \sum_{\langle i j\rangle \sigma}\left(c_{i \sigma}^{\dagger} c_{j \sigma}+\right.$ H.c. $)+U \sum_{i} n_{i \uparrow} n_{i \downarrow}+V \sum_{\langle i j\rangle} n_{i} n_{j}$,

which describes electrons moving on a lattice with a transfer integral $t$ and interacting via both on-site $(U)$ and nearest-neighbor Coulomb interactions $(V)$. We set the energy scale $t=1$. Studies of the EHM have mostly focused on bipartite lattices [2,6,7,9-11] (the Bethe lattice, or the square lattice in two dimensions), as these can naturally accommodate for charge ordering at a commensurate occupation of one charge per two lattice sites, corresponding to one quarter band filling. There are important cases, however, where the relevant lattice geometry is nonbipartite, adding frustration to the already rich phenomenology of correlated $\mathrm{CO}$. A notable example is the two-dimensional Wigner crystal itself, where charges arrange on a triangular pattern in order to minimize their electrostatic energy. How the analogy between the Wigner crystal transition and the Mott MIT is modified when one considers the appropriate triangular geometry [14] remains an open question, which was not addressed in Refs. [8,9]. A second example is the family of two-dimensional organic conductors $\theta$-(BEDT-TTF $)_{2} X[20,21]$, where BEDT-TTF is bis(ethylenedithio)tetrathiafulvalene, the molecular lattice is triangular, and the band filling is fixed by charge transfer to one hole per two molecular sites. In this framework, the EHM has been studied intensively to address the phenomenon of charge ordering, and fundamental differences between the square and triangular lattice have emerged. Most prominently, for isotropic $V$, on the triangular lattice, 
geometrical frustration prevents the twofold $\mathrm{CO}$ insulator characteristic of bipartite lattices, and a threefold $\mathrm{CO}$ metal is realized instead [cf. Fig. 1(b)] [12,13,16,17].

DMFT solution.-In its simplest form, DMFT maps a single-band Hubbard model onto an Anderson quantum impurity model in an effective metallic bath that is determined self-consistently. Allowing for charge ordering [6] with $N$-fold symmetry breaking implies solving $N$ interconnected (through hopping amplitudes) impurity models, one for each sublattice. Calling $G_{\alpha, \sigma}^{0}\left(i \omega_{n}\right)$ the Green's function of the $\alpha$ th electronic bath and $G_{\alpha, \sigma}^{\text {And }}\left(i \omega_{n}\right)$ that of the corresponding impurity problem, a local self-energy is extracted from the solution of the Anderson (And) model for each sublattice $\alpha$ via $\Sigma_{\alpha, \sigma}\left(i \omega_{n}\right)=G_{\alpha, \sigma}^{0}\left(i \omega_{n}\right)^{-1}-$ $G_{\alpha, \sigma}^{\text {And }}\left(i \omega_{n}\right)^{-1}$. The lattice Green's function within the dynamical mean-field approximation then reads $\mathbf{G}_{\sigma}\left(\mathbf{k}, i \omega_{n}\right)=\left[\left(i \omega_{n}+\mu\right) \mathbf{I}-\mathbf{E}_{H}-\mathbf{t}(\mathbf{k})-\boldsymbol{\Sigma}_{\sigma}\left(i \omega_{n}\right)\right]^{-1}$, where bold letters indicate $N \times N$ matrices in sublattice space, I is the identity matrix, $\mathbf{t}(k)$ is the Bloch matrix connecting the different sublattices, and $\mu$ is the chemical potential. Matsubara frequencies $\omega_{n}=(2 n+1) \pi / \beta$, with $\beta=200$ are used and $n_{\max }=2048$. Specializing to the threefold order realized on the triangular lattice, the matrix elements between sublattices $\alpha=A, B, C$ are $t_{A B}(k)=t_{B C}(k)=\phi_{k}, t_{A C}(k)=\phi_{k}^{*}$, having defined $\phi_{k}=$ $\sum_{i} e^{i \delta_{i} \cdot k}$ and $\delta_{i}(i=1,3)$ the vectors connecting an $A$ site to its three $B$ neighbors [see Fig. 1(b)]. $\mathbf{E}_{H}$ is a diagonal matrix whose elements $E_{H, \alpha}=3 \sum_{\gamma \neq \alpha} V\left\langle n_{\gamma}\right\rangle$ are, for each $\alpha$, the mean-field electrostatic potentials of charges on the nearest-neighboring sites. The problem is solved self-consistently by imposing the condition $(1 / L)$ $\sum_{\mathbf{k}} G_{\alpha \alpha, \sigma}\left(\mathbf{k}, i \omega_{n}\right)=G_{\alpha, \sigma}^{\text {And }}\left(i \omega_{n}\right)$, with $L$ the total number of lattice sites. We use Lanczos diagonalization as a
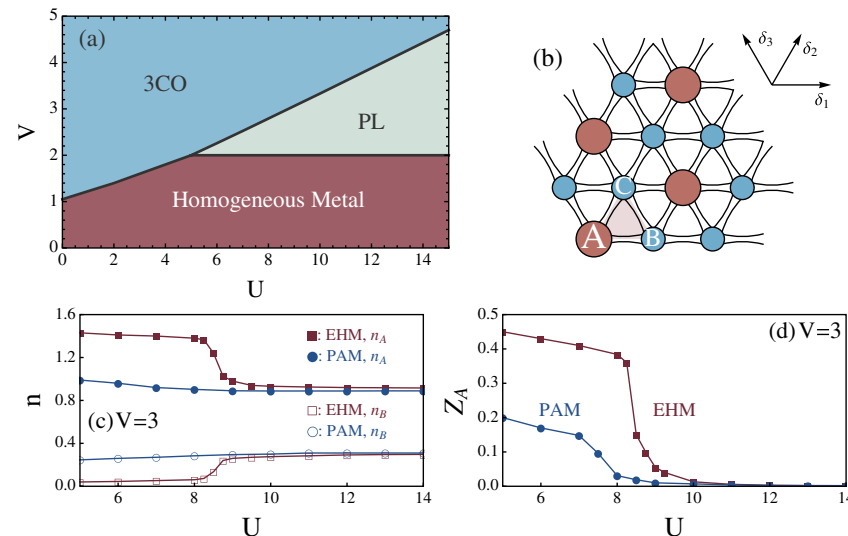

FIG. 1 (color online). Pinball liquid phase on the triangular lattice from DMFT. (a) The $U-V$ phase diagram of the EHM on the triangular lattice at quarter filling. (b) Sketch of the pinball phase with pins on the $A$ sublattice (red, large disks) and balls (blue, small disks) on the $B-C$ sublattice. The shaded area is the unit cell. Panels (c) and (d) are the charge occupations and quasiparticle weight $Z_{A}$ as a function of $U$. Blue lines show results for the PAM with $\Delta=4.5$. problem solver, taking $N_{s}=10$ sites for each quantum impurity problem [22]. The spectral density of electrons on the $\alpha$ sublattice is obtained from $A_{\alpha, \sigma}(\omega)=$ $-\operatorname{Im} G_{\alpha \alpha, \sigma}(\omega+i \eta) / \pi$, with $\eta=0.15$. We restrict our analysis to solutions where the $B$ and $C$ sublattices are equivalent. Practical calculations for a density $n$ of holes are performed by taking $n$ electrons per site and changing the sign of $t$ in Eq. (1).

The pinball liquid.-Figure 1(a) shows the phase diagram of the EHM obtained from DMFT on the triangular lattice at $n=1 / 2$ (quarter filling), which basically agrees with previous results obtained from exact diagonalization (ED) on small clusters [12,13]. Figures 2(a)-2(c) show representative spectral densities obtained in the three different phases. A transition from a homogeneous metal (HM) to a metallic $\mathrm{CO}$ state $(3 \mathrm{CO})$ is realized in the weakly correlated limit, $U \rightarrow 0$, upon increasing the intersite repulsion $V$, which is similar to what is found on bipartite lattices [7,9-11]. A large gap opens up in the spectrum due to the CO phenomenon [Fig. 2(c)], but the Fermi energy does not fall in the gap and the system remains metallic. At large $U$, instead, a different ordered metallic phase emerges, termed the pinball liquid [16,17], extending up to increasingly large values of $V$. In such a phase, quasilocalized charges (pins) coexist with itinerant carriers (balls) flowing in the honeycomb lattice formed by interstitial sites [see Fig. 1(b)]. Unlike the 3CO phase, where conduction arises from the charge-rich sites, in the PL it is the minority carriers (balls) who are responsible for the metallic behavior [see Fig. 2(b)]. The corresponding conduction band has a large width (comparable with that of the homogeneous metal itself) at
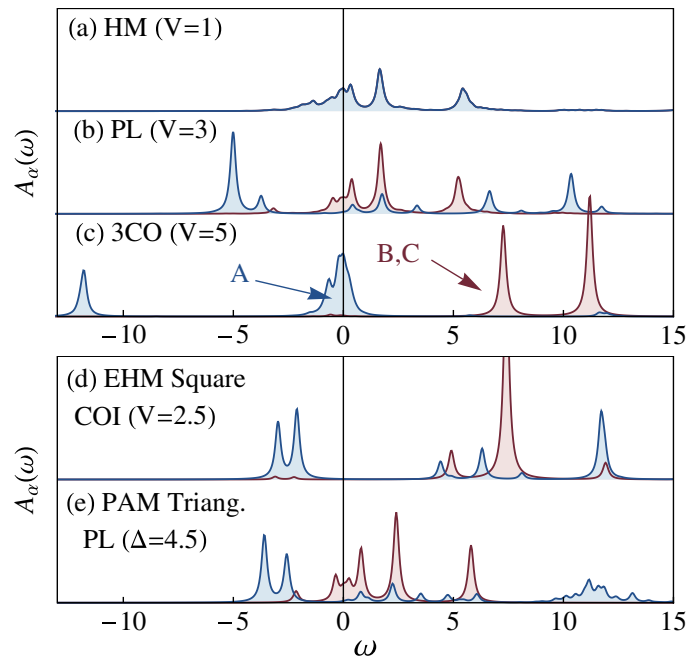

FIG. 2 (color online). Spectral densities $A_{A}(\omega)$ and $A_{B}(\omega)$ projected on the charge-rich and charge-poor sublattices, for $U=12$ in the EHM on the triangular lattice. (a) HM, (b) PL, and (c) $3 \mathrm{CO}$ phase. Panel (d) shows the same quantity in the $\mathrm{CO}$ insulating phase on the square lattice. Panel (e) is the spectral density calculated for the PAM at $\Delta=4.5$. The vertical line denotes the Fermi energy. 
all values of $V$, and does not scale with $\propto t^{2} / V$ as in the $3 \mathrm{CO}$ phase. Note that the pinball phase is absent on the square lattice, where the system is $\mathrm{CO}$ and insulating (COI) at sufficiently large $U$ [7,9-11]; cf. Fig. 2(d).

The origin of the pinball phase can be best understood as follows. At large $V$ and small $U$, the $3 \mathrm{CO}$ phase has a density $n_{A} \rightarrow 3 / 2$ on the charge-rich sites and $n_{B} \rightarrow 0$ on the charge-poor sites. Increasing $U$ tends to suppress the double occupancy and eventually when $U \gtrsim 3 V$, the local Coulomb energy cost is avoided by leaving only one charge per $A$ site (a pin, $n_{A} \simeq 1$ ). The excess charge density is then transferred to the charge-poor sites (balls, $n_{B} \simeq 1 / 4$ ). This transition, shown in Fig. 1(c), occurs between two metallic phases, which only differ on the amount of charge transferred. This transition is of a different type from the metal-to-COI transition [11] on the square lattice, which displays phase coexistence as in the pure Mott metalinsulator transition. We find that the two charge species behave very differently: pins form an almost half-filled system with a marked Mott behavior due to strong on-site Coulomb interactions, in opposition to the balls which, owing to their low concentration, are, in principle, protected from them. The spectral function for the $B, C$ sublattices indeed shows a wide band crossing the Fermi energy, while the $A$ sublattice shows typical features of strongly correlated systems: the spectral weight is mostly located in two separate regions away from the Fermi level-the lower and upper Hubbard bands separated by an energy $U$-while the weight at the Fermi energy is strongly suppressed. This can be quantified via the quasiparticle weight of the correlated pins, $Z_{A}=$ $\left[1-\partial \operatorname{Im} \Sigma_{A}(\omega) / \partial \omega\right]_{\omega=0}^{-1}$, which is depicted in Fig. 1(d). It is large in the conventional $3 \mathrm{CO}$ phase at low $U$, and drops drastically upon entering the pinball liquid (here at $U=8.5$ ). This drop occurs when the $A$ sublattice concentration spontaneously adjusts to $n_{A} \simeq 1$ [Fig. 1(c)]. Within the accuracy of our method, a small nonvanishing quasiparticle weight persists at all $U$ [23]. Since the filling of the correlated hybrid band is never exactly an integer, the results indicate that the pins do not undergo a full Mott transition, but remain in a strongly correlated Fermi liquid state. This contrasts with the situation encountered on bipartite lattices [11], where the electron concentration in the correlated band is automatically fixed to half-filling once the Fermi energy falls inside the gap, resulting in a true Mott transition for $U \gtrsim W$ (the bandwidth) and $V \gtrsim 2$.

Periodic Anderson model and emergent heavy fermions.To get further insight into the effects of electronic correlations we now map the problem into a PAM. This is achieved by introducing creation operators $a_{i}^{+}, b_{i}^{+}$, and $c_{i}^{+}$respectively on the $A, B$, and $C$ sublattices and diagonalizing the bands of the honeycomb lattice formed by $B$ and $C$ sites (tight binding on graphene). Rewriting $\phi_{k}=$ $\sum_{i} e^{i \delta_{i} \cdot k}$ as $E_{k} e^{i \theta_{k}}$, we can define $B-C$ subspace spinor operators $\gamma_{k, \pm}^{+}=\left(e^{-i \theta_{k} / 2}, \pm e^{i \theta_{k} / 2}\right) / \sqrt{2}$, whose energies are $\epsilon_{k, \pm}= \pm E_{k}$ (the origin is fixed at the Dirac point). Dropping the Coulomb interaction on the conduction bands leads to

$$
\begin{aligned}
H_{\mathrm{PAM}}= & \sum_{i \sigma} \epsilon_{A} a_{i \sigma}^{\dagger} a_{i \sigma}+\sum_{k \sigma \alpha} \epsilon_{k, \alpha} \gamma_{k \alpha \sigma}^{+} \gamma_{k \alpha \sigma} \\
& +\sum_{i k, \alpha}\left(V_{k, \alpha} e^{i k R_{i}} a_{i \sigma}^{\dagger} \gamma_{k \alpha \sigma}+\text { H.c. }\right) \\
& +U \sum_{i} a_{i \uparrow}^{\dagger} a_{i \uparrow} a_{i \downarrow}^{\dagger} a_{i \downarrow},
\end{aligned}
$$

where the hybridization between the conduction bands and the localized level is determined by $V_{k,-}=$ $i \sqrt{2} E_{k} \sin \left(3 \theta_{k} / 2\right)$ and $V_{k,+}=\sqrt{2} E_{k} \cos \left(3 \theta_{k} / 2\right)$. The energy of the localized level is set by the charge transfer gap, $\epsilon_{A}=-\Delta$, which originates from the electrostatic interaction between charges on different sublattices. The pinball phase studied in the preceding paragraphs corresponds to $\Delta=$ $3 \mathrm{~V} / 2$ and is therefore in the charge transfer regime $U \gg \Delta$ [because $U>3 V \gg 3 V / 2$, cf. Fig. 1(a)]. The quantities $n_{\alpha}$, $Z_{A}$, and $A_{\alpha}(\omega)$ calculated in the PAM are compared with the EHM results in Figs. 1(c), 1(d), 2(a), and 2(b), showing a consistent agreement in the pinball liquid phase.

The nature of the coupling between pins and balls is now conveniently analyzed based on the low energy effective PAM, varying the number of electrons per unit cell $n_{\text {cell }} \in$ $[0,3]\left(n_{\text {cell }}=3 n\right)$ for a fixed $\Delta=4.5$. This interval includes the region of stability of the pinball in the original EHM [18], i.e., $1<n_{\text {cell }}<2$. The density of states (DOS) of $H_{\text {PAM }}$ in the noninteracting limit $U=0$ is shown in Fig. 3(a). Hybridization broadens the localized pin level originally at $\epsilon_{A}=-\Delta$ into a dispersive band, and also affects the dispersion of the conduction bands, which is no
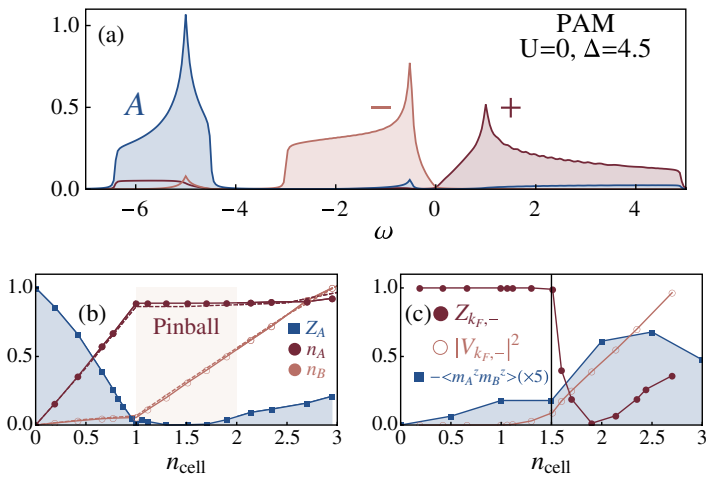

FIG. 3 (color online). Strong quasiparticle renormalization in the pinball liquid phase and antiferromagnetic Kondo correlations. (a) DOS of the noninteracting PAM $(U=0)$ for $\Delta=4.5$. Different colors correspond to a projection on the nonhybridized bands: pins (blue), - (orange), and + (red). (b) $A / B$ sublattice densities (full lines = DMFT, dashed lines = ED) and pin quasiparticle weight (DMFT) and (c) hybridization, quasiparticle renormalization (DMFT), and magnetic correlations (ED) as a function of the filling $n_{\text {cell }}$ at $U=12$. 
longer symmetric around the Dirac point $\omega=0$. Because $\epsilon_{A}<0$, pins are populated first and $n_{A} \simeq n_{\text {cell }}$ until $n_{\text {cell }}=1$ as depicted in Fig. 3(b). For $n_{\text {cell }}>1$, the $A$ sublattice is filled with $\simeq 1$ electron per site (the maximum allowed by the large $U$ ) and the remaining electrons move to the conduction band. Correspondingly, the Fermi energy moves from the bottom of the (-) band to the Dirac point, reached at $n_{\text {cell }}=3$. Interestingly, $Z_{A}$ [blue squares in Fig. 3(b)] is strongly suppressed in the whole pinball phase [hatched region in Fig. 3(b)]. Following Refs. [25,26], we define the effective self-energy for conduction electrons as

$\Sigma_{k, \pm}\left(i \omega_{n}\right)=\frac{\left|V_{k, \pm}\right|^{2}}{i \omega_{n}+\mu-\epsilon_{A}-\Sigma_{A}\left(i \omega_{n}\right)-\frac{\left|V_{k,+}\right|^{2}}{i \omega_{n}+\mu-E_{k}}}$,

which accounts for hybridization, and includes crucial interaction effects arising indirectly via the Coulomb repulsion of localized charges. Based on Eq. (3), we evaluate the quasiparticle renormalization $Z_{k, \pm}=$ $\left[1-\partial \operatorname{Im}\left(\Sigma_{k, \pm}\right) / \partial \omega\right]_{\omega=0}^{-1}$ of the balls. Note that $V_{k,-}$ vanishes for wave vectors $k$ forming an angle $\pi / 6[\pi / 3]$ with the $x$ axis, leading to cold spots where conduction and localized electrons are perfectly decoupled and $Z_{k,-}=1$ identically. In Fig. 3(c) we report instead $Z_{k,-}$ in the directions of maximum hybridization on the Fermi surface (hot spots), for which a sharp drop is found at $n_{\text {cell }}=3 / 2$. This is attributed to an increase in the hybridization with the correlated pins occurring around this filling (see also $\left.\left|V_{k,-}\right|^{2}\right)$. On the same figure is also reported the interspecies magnetic correlation $\left\langle m_{z}^{A} m_{z}^{B}\right\rangle=\left\langle\left(n_{A \uparrow}-n_{A \downarrow}\right) \times\right.$ $\left.\left(n_{B \uparrow}-n_{B !}\right)\right\rangle$ calculated by ED on a 12-site cluster (we use ED because intersite correlations cannot be addressed in the single site DMFT scheme). As shown in Fig. 3(c), the strong renormalization of the metal is accompanied by a buildup of antiferromagnetic correlations, corresponding to a screening of the localized moments by the conduction electrons. From our data we conclude that pins and balls are nearly decoupled for $n_{\text {cell }} \leq 3 / 2$, while a strong Kondo coupling between the two fluids, accompanied by a strong renormalization of the conduction electrons, arises at larger concentrations.

Wigner crystal in the 2DEG.-According to the lattice analogy proposed in Refs. [8,9,14], the existence of pronounced short range order close to the MIT enables a mapping of the continuous 2DEG to our model $H_{\text {PAM }}$ at $n_{\text {cell }}=1$. We can then associate $\Delta \propto r_{s}$ [14], where $r_{s}$ is the ratio of Coulomb to kinetic energy in the electron gas. The case $\Delta=4.5$ analyzed in Fig. 3(b) then corresponds, for $n_{\text {cell }}=1$, to a $2 D E G$ well into the Wigner crystal phase: the system is insulating, as both $Z_{A}$ and the DOS at the Fermi energy (not shown) vanish at this point. Following Ref. [14], the quantum melting of the Wigner crystal upon reducing $r_{s}$ can be identified with a closing of the gap between the pin and (-) bands [Fig. 3(a)] occurring below a critical $\Delta_{c}$. From our DMFT results, this happens at
$\Delta_{c} \simeq 3.7$ for $U=12$. Although a detailed study of the MIT of the 2DEG is beyond the scope of this work, we note that a metallic state can also be achieved at $\Delta>\Delta_{c}$, by doping away from $n_{\text {cell }}=1$. The resulting metal is very different when doping holes into the pin band $\left[n_{\text {cell }}=1+\delta\right.$ with $\delta<0$, leading to $Z_{A} \propto|\delta|$, cf. Fig. 3(b)] or electrons into the conduction band ( $\delta>0$, with $Z_{A}$ jumping sharply to a small but finite value). The latter situation actually corresponds to the self-doping instability proposed in Ref. [8]. If this scenario were realized, the metal on the verge of Wigner crystallization would actually be a pinball liquid itself, indicating an appealing connection between the physics of the 2DEG and the emergence of heavy fermion behavior.

Concluding remarks.-The combination of electronic correlations and geometrical frustration on charge ordered triangular lattices leads to an exotic pinball liquid state in which localized and itinerant electrons coexist. Based on the close analogies with heavy fermion systems [19], pointed out via an explicit mapping of the electronic problem onto the periodic Anderson model, non-Fermi liquid behavior should occur in such a phase as a consequence of the scattering of the itinerant electrons off the localized moments of the pins. The scenario proposed here could be of relevance to several correlated materials exhibiting charge ordering on triangular lattices such as the organic conductors $\theta$-(BEDT-TTF $)_{2} X$ [27], transitionmetal dichalcogenides [3-5], $\mathrm{AgNiO}_{2}$ [28] and ${ }^{3} \mathrm{He}$ bilayers [29], as well as to the interaction-driven MIT in the 2DEG $[8,9,11]$.

J.M. acknowledges financial support from MINECO (MAT2012-37263-C02-01). This work is supported by the French National Research Agency through Grant No. ANR-12-JS04-0003-01 SUBRISSYME.

[1] M. Imada, A. Fujimori, and Y. Tohura, Rev. Mod. Phys. 70, 1039 (1998).

[2] H. Seo, J. Merino, H. Yoshioka, and M. Ogata, J. Phys. Soc. Jpn. 75, 051009 (2006).

[3] P. Fazekas and E. Tosatti, Philos. Mag. B 39, 229 (1979).

[4] L. Perfetti, P. Loukakos, M. Lisowski, U. Bovensiepen, H. Berger, S. Biermann, P. Cornaglia, A. Georges, and M. Wolf, Phys. Rev. Lett. 97, 067402 (2006).

[5] B. Sipos, A.F. Kusmartseva, A. Akrap, H. Berger, L. Forró, and E. Tutiš, Nat. Mater. 7, 960 (2008).

[6] R. Pietig, R. Bulla, and S. Blawid, Phys. Rev. Lett. 82, 4046 (1999).

[7] J. Merino, Phys. Rev. Lett. 99, 036404 (2007).

[8] S. Pankov and V. Dobrosavljević, Phys. Rev. B 77, 085104 (2008).

[9] A. Camjayi, K. Haule, V. Dobrosavljević, and G. Kotliar, Nat. Phys. 4, 932 (2008).

[10] C. Li and Z. Wang, Phys. Rev. B 80, 125130 (2009)

[11] A. Amaricci, A. Camjayi, K. Haule, G. Kotliar, D. Tanasković, and V. Dobrosavljević, Phys. Rev. B 82, 155102 (2010). 
[12] L. Cano-Cortés, J. Merino, and S. Fratini, Phys. Rev. Lett. 105, 036405 (2010).

[13] L. Cano-Cortés, A. Ralko, C. Fevrier, J. Merino, and S. Fratini, Phys. Rev. B 84, 155115 (2011).

[14] Z. Lenac and M. Šunjić, Phys. Rev. B 52, 11238 (1995).

[15] M. M. Radonjić, D. Tanasković, V. Dobrosavljević, K. Haule, and G. Kotliar, Phys. Rev. B 85, 085133 (2012).

[16] M. Kaneko and M. Ogata, J. Phys. Soc. Jpn. 75, 014710 (2006).

[17] C. Hotta and N. Furukawa, Phys. Rev. B 74, 193107 (2006).

[18] C. Hotta and N. Furukawa, J. Phys. Condens. Matter 19, 145242 (2007).

[19] P. Gegenwart, Q. Si, and F. Steglich, Nat. Phys. 4, 186 (2008).

[20] H. Kino and H. Fukuyama, J. Phys. Soc. Jpn. 65, 2158 (1996).

[21] H. Mori, S. Tanaka, and T. Mori, Phys. Rev. B 57, 12023 (1998).
[22] This is sufficient to find a reliable projection to the Anderson model through conjugate gradient minimization of $\sum_{n}\left|G_{\alpha \sigma}^{0}\left(i \omega_{n}\right)-G_{\alpha \sigma}^{0 \text { And }}\left(i \omega_{n}\right)\right|$, for each $\alpha$ sublattice.

[23] Because of finite discretization, a small $Z_{\alpha}$ obtained from the smallest Matsubara frequency could be consistent with a Mott insulator [24].

[24] N. Bluemer, Ph.D. thesis, Universitaet Augsburg, 2002.

[25] A. C. Hewson, The Kondo Problem to Heavy Fermions (Cambridge University Press, Cambridge, England, 1993).

[26] A. Amaricci, L. de Medici, G. Sordi, M. J. Rozenberg, and M. Capone, Phys. Rev. B 85, 235110 (2012).

[27] M. Hirata, K. Miyagawa, K. Kanoda, and M. Tamura, Phys. Rev. B 85, 195146 (2012).

[28] E. Wawrzyńska, R. Coldea, E. Wheeler, I. Mazin, M. Johannes, T. Sörgel, M. Jansen, R. Ibberson, and P. Radaelli, Phys. Rev. Lett. 99, 157204 (2007).

[29] M. Neumann, J. Nyéki, B. Cowan, and J. Saunders, Science 317, 1356 (2007). 